Special Issue of the 7th International Advances in Applied Physics and Materials Science (APMAS 2017)

\title{
Design of Microcontroller Controlled YAG Laser Device Cooling System using Thermoelectric Materials
}

\author{
M. YAGCI* \\ Necmettin Erbakan University, Faculty of Engineering and Architecture, \\ Department of Electrical and Electronics Engineering, Konya, Turkey
}

\begin{abstract}
Cooling in solid state lasers is an important factor. The laser rod and the flashlight are cooled by force. The waste heat generated throughout the laser device must be removed from the device. Small powerful systems are air-cooled or nitrogen. The gases do not provide a good heat energy transfer. However, gas cooling is sufficient for low power pulsating systems. Most solid state lasers use liquid-cooled systems. Water is also used as the cooling fluid. In this study, the temperature of the cooling water was controlled by a PIC controlled thermoelectric system and cooling was performed.
\end{abstract}

DOI: 10.12693/APhysPolA.134.335

PACS/topics: Nd:YAG laser, laser rod, micro controller, thermoelectric material (Peltier), temperature control

\section{Introduction}

In solid state lasers, the laser rod is driven by the flash lamp to achieve population tie-up. When flash lamp arc is obtained, the heat released should be removed from the medium. If the laser rod heats up excessively, the glass laser rod used to obtain the laser medium may break and damage or deteriorate the quality of the laser beam [1-3].

In this article, PIC controlled thermoelectric system is designed for solid state laser system. Thermoelectric modules (the Peltier module) used for cooling purposes are controlled by PIC16F84 microcontroller manufactured by microchip company.

With the help of the microcontroller, the temperature of the laser cooling water is proportionally controlled with the aim of being more sensitive at the desired level. The block representation of the system is shown in Fig. 1.

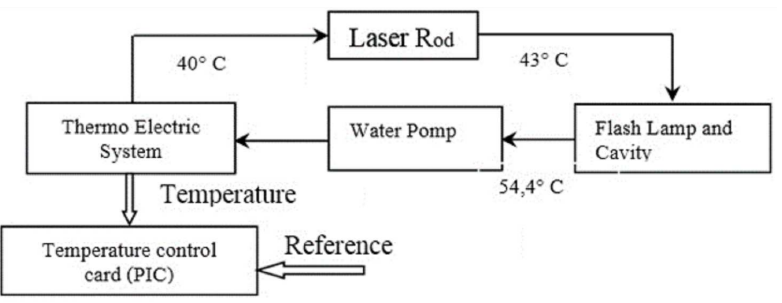

Fig. 1. Block representation of the laser cooling system.

\section{Neodymium:YAG (Nd:YAG)}

The chemical formula of YAG is $\mathrm{Y}_{3} \mathrm{Al}_{5} \mathrm{O}_{12}$. The thermal conductivity of YAG is 10 times higher than glass. It has a high melting point and a strong and solid structure.

*e-mail: myagci@konya.edu.tr
The pure YAG crystal is apparently similar to sapphire. When neodymium is added infrequently, the color of the laser rod YAG appears to be slightly purple. Neodymium atoms in the crystal structure are ionized into triplets as if they were chromium atoms in the same ruby laser bar. Figure 2 shows the absorption spectrum of Nd:YAG. YAG's absorption lines consist of sharp peaks and one near band. The two major pumping band regions are $730-760 \mathrm{~nm}$ and $790-820 \mathrm{~nm}$. This is very suitable for optically pumping because these bands are near infrared. Optical energy is absorbed in short wave lengths [3-6].

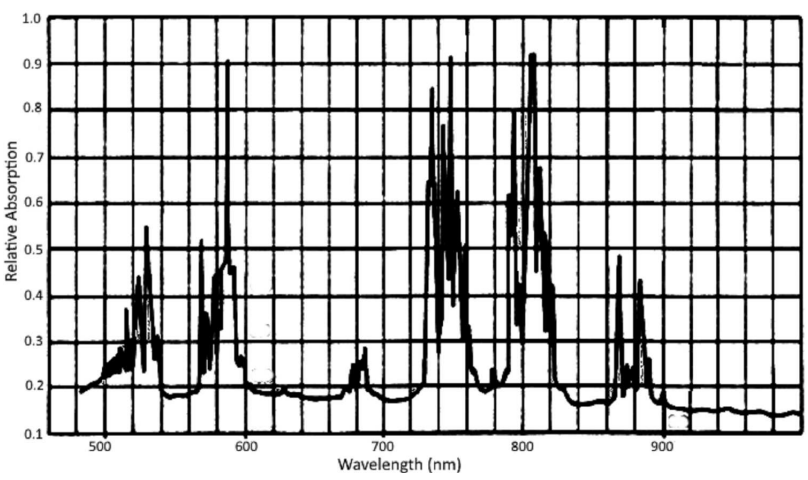

Fig. 2. Absorption spectrum of Nd:YAG..

Figure 3 shows a diagram of the energy levels of Nd:YAG absorption photons. The energy of the neodymium atoms will increase and rise from the pumping bands. The lifetime of an electron in this band is 10-8 s. During this time the atom will rapidly fall to the upper laser level without any propagation.

The fluorescence lifetime at the upper laser level $\left({ }^{4} \mathrm{~F}_{3 / 2}\right)$ lasts $0.3 \mathrm{~ms}$. With the formation of the population tip, the laser is formed and the atom falls to the lower laser level. The lower laser level is very close to the ground level and the excited atoms quickly return to the ground level without a radiation pulse. Transmitted atoms emitted without radiation emit heat energy. 


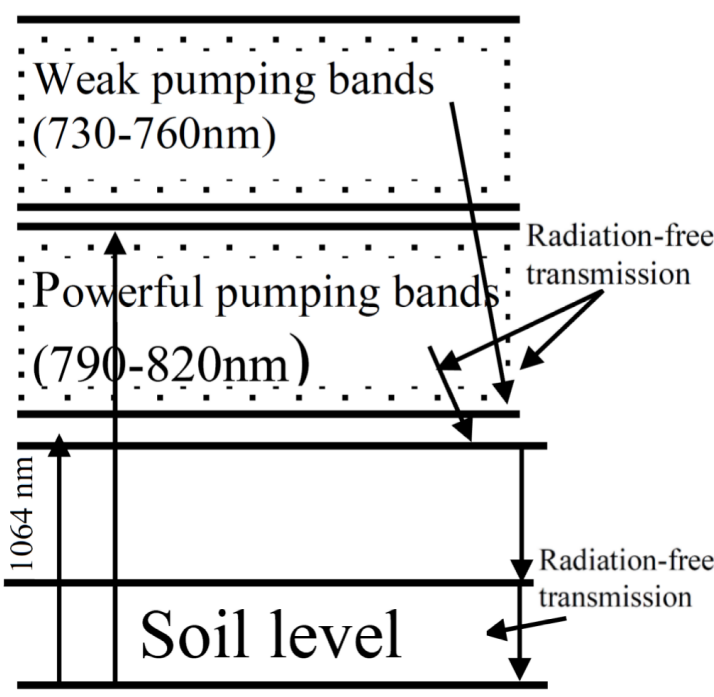

Fig. 3. Energy levels of the Nd:YAG laser.

The heat energy that is released here is also absorbed by the optical cavity and the laser rod. If the temperature of the laser bar becomes too high, the atoms will cause the population to cross between the lower level laser and the ground level with the effect of heat.

This will disrupt the ordering required for the laser and prevent the formation of a laser beam. Because of this, it is very important to keep the Nd:YAG laser bar at a certain temperature.

It is necessary to cool the bar in these types of lasers because of the constantly open thermal energy. Since the thermal conductivity of $\mathrm{Nd}$ :YAG is very good, it can be efficiently cooled with pure water as cooling fluid. Nd:YAG lasers can be operated in continuous mode by cooling. As a solid-state laser that can be operated in continuous mode, the YAG industry is widely used. The laser bar to be operated in continuous wave mode has a diameter of 1 to $4 \mathrm{~mm}$ and a length of 2.5 to $15 \mathrm{~cm}$. Small diameter ones are chosen instead of large diameter laser bars for easy cooling. The laser rod is placed in a quartz or protective glass sheath to protect the coating material in the water. The cooling of the laser bar is done by flowing liquid through the process. The laser rod element is mounted in the optical cavity with special sealing elements to prevent liquid leakage and thus the damage of the flash lamp.

\section{Cooling systems}

The cooling system is one of the most important critical subsystems of the laser device. The small power lasers are cooled by the open loop cooling systems with water flowing as coolant liquid through the laser rod. Large powerful laser systems are cooled with closed loop cooling systems. In the mentioned systems, antifreeze water mixture is used as cooling fluid. The heat of the cooling fluid is transferred from water-to-water or water-to-air to another medium using heat exchangers.
The water flow rate for the cooling system and the total temperature rise in the cooling water are calculated as follows [3]:

$$
P_{P L T}=P_{i n} \eta_{P S} \eta_{F L} \eta_{R G}
$$

where $P_{P L T}$ - total power absorbed by the laser rod, $P_{i n}$ — input power of flash lamp, $\eta_{P S}$ - efficiency of power supply, $\eta_{F L}$ - the wavelength at which the laser rod can absorb efficiency, $\eta_{R G}$ - efficiency of optical covity.

Amount of heat to be removed from the laser rod

$P_{L R P}=P_{P L T}-P_{O L}$

amount of power to be removed from the laser rod, $P_{L R P}$ - laser output power.

$$
H_{L R P}=\frac{P_{L R P}}{4.18}
$$

$1 \mathrm{cal}=4.18 \mathrm{~J}, 1 \mathrm{~W}=1 \mathrm{~J} / \mathrm{s}), H_{L R P}$ - amount of energy to be removed from the laser rod.

$$
V_{F R}=\frac{H_{L R P}}{3}
$$

coolant flow rate.

$$
H_{T O T}=\frac{P_{T O T}}{4.18}
$$

total amount of energy to be cooled. $P_{T O T}-$ total amount of power to be cooled, $P_{P S L}$ - power supply losses.

$$
H_{T R}=\frac{H_{T O T}}{V_{F R}}
$$

amount of increase in temperature.

\section{Thermoelectric element}

Thermoelectric elements (the Peltier element) have acquired a commercial characteristic after 1960's, today in different countries, in laboratories, portable coolers/heaters, in military areas, and in envy [7, 8].

If some advantages of thermoelectric module are to be listed as materials:

- Its size is small and its weight is low;

- Reliable, quiet and unstable because of its semiconductor nature;

- Average life is over $200000 \mathrm{~h}$ (> 22 years);

- Can perform temperature control effectively;

- The electrical noise is low;

- It works with DC voltage;

- Heating and cooling can only be selected by reversing the direction of current.

If a current $I$ is passed from a closed circuit consisting of two different materials such as $\mathrm{A}$ and $\mathrm{B}$, the amount of heat that is absorbed or removed from the unit is proportional to the current $I$ and can be calculated as [9]:

$$
Q=\pi_{A B} I
$$

where $Q$ - amount of heat transferred per unit time [W], $\pi_{A B}$ - the Peltier constant for materials A and B [V], $I$ - direct current $[\mathrm{A}]$. 
Cold and hot surfaces are the natural result of heat transfer which is completely formed by the Peltier effect. That is, the heat is transferred from the cold surface to the hot surface by the Peltier effect, and no heat is produced. Semiconductor physics studies show that a thermoelectric cooler is a heat pump that uses the energy levels of electrons to transfer heat. Electrons pass through the junction of two different thermoelectric materials, they are undergoing an energy absorber or emitter change. While the current flows from the $p$-type semiconductor with the lower energy level to the $n$-type semiconductor with the higher energy level, the electrons leave on the energy hot surface they receive from the cold surface. The externally applied electrical power provides the energy required for the electrons to move within the system, so that the electrons that are moving move heat while traveling between varying energy levels are carrying heat. If the external electrical power is increased, the amount of heat transfer between the cold surface and the hot surface will increase as the number of moving electrons increases. If the direction of the current is reversed, this operation will reverse and the hot and cold surfaces will shift between themselves.

The element consisting of two different materials in Fig. 4 is called a couple. The $N$-type thermoelectric material has a negative Seeback coefficient and an electron excess. The $P$-type thermoelectric material has a positive Seeback coefficient and electron deficiency. The direction of current is in the known direction, and the flow direction of the electrons is reversed. Although thermoelectric material has four connection points between copper sheets, there are only two thermoelectric junctions, one at the top and the other at the bottom. When heat is absorbed from the upper (or colder) surface, it is released from the lower (or hot) surface.

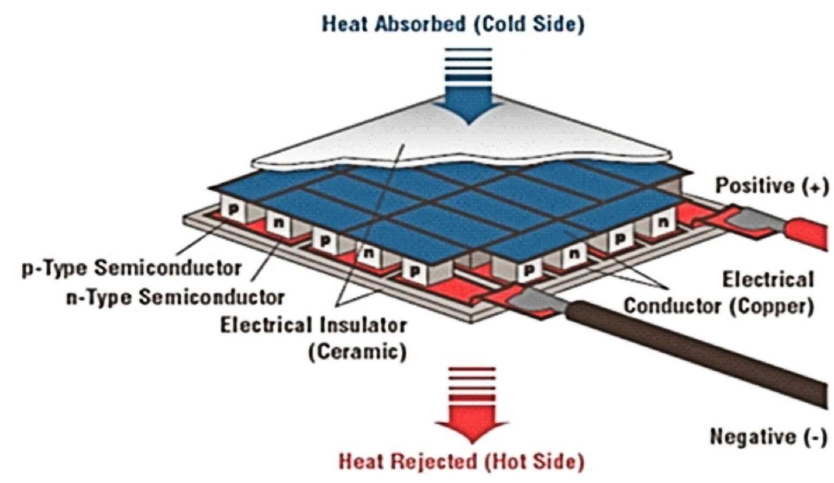

Fig. 4. Structure of thermoelectric module.

\section{Control system}

The temperature control system used is shown in Fig. 5. The temperature signal sensed by the sensor is compared with the set value after the ambient temperature compensation has been performed, after pass- ing through an amplifier circuit. By taking the difference between the two, the error value or difference value is found.

If this value is positive, the process is below the set value. If negative, it is above the process set value. If the difference is zero, the process is at the set value. The difference value passes through the proportional control circuits and arrives in the appropriate output form. If the difference value is zero then the proportional output is $50 \%$. It means that it works at set value. It is difficult to keep the output at $50 \%$ and keep the process at full set value.

The most prominent characteristic of proportional control is that there is a temperature change until the equilibrium state is reached, and even a certain difference between the temperature value and the set value remains. The difference between the set point and the temperature at which the system is sitting and remains constant is called off-set.

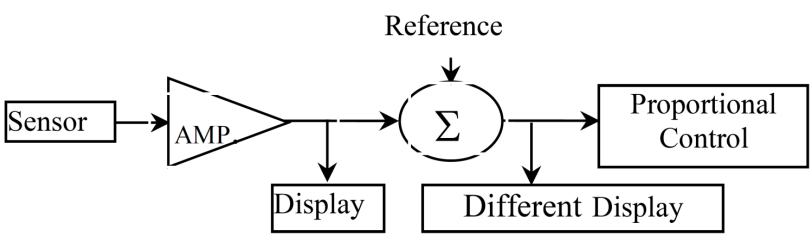

Fig. 5. Proportional control block diagram.

The proportional band may be reduced to reduce the off-set, but in this case the system approaches the on-off control and oscillations occur around the set point. Considering that the off-set is large in the wide proportional band, the optimum band should be selected.

The temperature rises, it sits around the set point several times after it is released, then it comes to a fixed temperature difference above or below the set value. Off-set can be positive or negative $[10,11]$.

\section{Conclusion}

In this study, an innovative cooling system for small power laser systems was designed and realized by using the Peltier element to cool the laser bar and the flash lamp forcing in solid state lasers.

Since the cooling fluid is not circulated in the system, the adverse effect of the liquid on the laser devices has been eliminated. As the cooling capacities of the Peltier elements are low, the surface area of the laser cavity is enlarged and the capacity of the cooling system is increased by placing a large number of the Peltier elements on this cavity.

This developed system is thought to be suitable for small powerful lasers. However, because of the low capacity of the Peltier elements for large power lasers, it is not feasible to use it efficiently without making a change to the existing cavity system. 


\section{Acknowledgments}

This study was supported by Necmettin Erbakan University Scientific Research Projects Office.

\section{References}

[1] J.E. Harry, Industrial Lasers and Their Applications, McGraw-Hill, England 1974.

[2] J.F. Ready, LIA Handbook of Laser Materials Processing, Springer, Berlin 2001.

[3] N. Tarakcioglu, M. Ozcan, Lasers and Material Processing, Atlas, Istanbul 2004.

[4] M. Ozcan, Ph.D. Thesis, Selcuk University, Institute of Science and Technology, Konya 2003.

[5] C. Gravis, Progr. Quant. Electron. 45, 3 (2016).
[6] R. Xu, Q. Su, Modern Inorganic Synthetic Chemistry, 2nd ed., Elsevier, 2017.

[7] A.F. Ioffe, Poluprovodniki, Russian Academy of Sciences Publishing House, Moscow-Leningrad 1960, (in Russian).

[8] D.H. Haynes, W.P. Monaghan, Military Medicine 153, 86 (1988).

[9] K. Matsubara, in: Proc. 21st Int. Conf. on Thermoelectrics, Long Beach 2002, p. 418.

[10] N.A. Anderson, Instrumentation for Process Measurement and Control, Chilton Book, 1980.

[11] A.L. Reenstra, IEEE Trans. Ind. Appl. 1, 58 (1973). 